\title{
Avaliação das tecnologias de softwares existentes para a Inclusão Digital de dEficientes visuais através da utilização de Requisitos de qualidade.
}

\author{
Clóvis da Silveira - Centro Universitário Feevale - csilveira@feevale.br \\ Regina de Oliveira Reidrich - Centro Universitário Feevale - rheidrich@feevale.br \\ Patrícia Brandalise Scherer Bassani - Centro Universitário Feevale - \\ patriciab@feevale.br
}

\begin{abstract}
Assessment of softwares tecnologies to the digital inclusion of visual disable through using of quality requirements.
\end{abstract}

\begin{abstract}
This article show the importance the technology of reader softwares web to inclusion and acessibity to visual deficience person. Digital Inclusion and acessibility don't meaning just have on program to try use the computer, but one software technology to attend all necessity of your usuary with visual deficience. To comprehend that and how to evaluate this necessities this article show a research to base in a model of quality software NBR ISO/IEC 9126, where across elaboration questionary, five usuary with visuais deficience already used at least one this technologies and they and to evaluate reader softwares Virtual Vision 5.0, Jaws 8 e NVDA

.Keywords: deficient visual, Digital Inclusion, Model of Quality
\end{abstract}

Resumo. Este artigo apresenta a importância das tecnologias de softwares leitores de telas para a inclusão e acessibilidade de pessoas portadoras de deficiência visual. Inclusão digital e acessibilidade, não significa apenas ter um programa para tentar usar o computador, mas sim uma tecnologia de software que atenda todas as necessidades dos usuários dEficientes visuais. Para compreender o que e como avaliar essas necessidades do usuário o presente artigo apresenta uma pesquisa baseada no modelo de qualidade de software NBR ISO/IEC 9126 onde, a partir de um questionário, cinco usuários dEficientes visuais que já utilizavam ao menos uma dessas tecnologias avaliou os softwares leitores Virtual Vision 5.0, Jaws 8 e NVDA.

Palavras Chaves: Dificientes visuais, Inclusão digital, Requisitos de Qualidade.

\section{Introdução}

Ultimamente temos observado, o grande avanço das tecnologias de software para a inclusão de dEficientes visuais. No entanto, essas "tecnologias" passam despercebidas e pouco exploradas por seu público. Por outro lado, algumas escolas, prefeituras, associações e universidades oferecem esses treinamentos gratuitamente.

Mas a acessibilidade a esses softwares continua sendo a realidade de poucas pessoas pelos mais diversos motivos, seja desde a falta de um local para realizar o treinamento, até uma pessoa para levar o dEficiente1 visual até o local dos cursos, além disso, muitos dos familiares mantém essas pessoas dentro de casa, impedindo sua inclusão junto as

\footnotetext{
1 Escrevemos dEficiente desta forma conforme Heidrich (2004) para ressaltar a eficiência na deficiência do ente "ser".
} 
tecnologias existentes, pois acham que o dEficiente visual não é capaz de realizar algo porque é "cego".

Antes mesmo de se falar em softwares para dEficientes visuais tem-se que entender um pouco sobre a dEficiência visual. As pessoas com dificuldades visuais são classificadas em dois grupos principais: cegos e com visão parcial ou reduzida. Mesmo desta forma, os dois grupos se enquadram no termo cegueira.

A cegueira reúne indivíduos com vários graus de perda de visão, podendo ser a ausência total de visão até a perda da projeção de luz. Ela não significa, necessariamente, total incapacidade para ver, mas sim, prejuízo dessa capacidade. O portador de visão parcial ou reduzida tem condições de indicar projeção de luz até o grau em que a redução da acuidade visual interfere ou limita seu desempenho.

Para a inclusão digital de pessoas com esse tipo de dEficiência, temos alguns softwares leitores de telas que permitem a utilização do computador, possibilitando a utilização do sistema operacional Windows, aplicativos de textos, planilhas eletrônicas, acesso a correio eletrônico e internet.

Nessa perspectiva, iremos realizar uma avaliação dos softwares leitores de telas Virtual Vision 5.0, Jaws 8.0 e NVDA, através de um método de avaliação de qualidade de software, com o objetivo de identificar qual é o melhor software leitor de telas para seu público a partir de uma avaliação prática realizada por cinco usuários dEficientes visuais, que já tem conhecimento de software leitor de tela.

\section{Inclusão e acessibilidade}

Para que o processo de inclusão educativa ocorra de uma forma efetiva, é necessário que haja uma união entre todos os membros da comunidade escolar, a fim de que possam atuar no processo de transformação desta escola.

Diferenciar o ensino é organizar as interações e atividades de modo que cada aluno se defronte, constantemente, com situações didáticas que lhes sejam as mais fecundas (PERRENOUD, 2005, p. 36).

A diferenciação não é um método, mas exige uma grande investigação sobre atividades e situações de aprendizagem que sejam significativas e mobilizadoras, levando em conta as diferenças pessoais e culturais de cada aluno. A inclusão é uma possibilidade que se abre para qualificar a educação aos alunos com necessidades educativas especiais e aos alunos comuns.

Contribuindo com este pensar, Beyer (2005, p. 67) afirma que "a inclusão propõe um único sistema educacional de qualidade para todos os alunos, com ou sem deficiência e com ou em outros tipos de condição atípica”. Assim, a educação inclusiva depende não só da capacidade do sistema escolar (diretor, professores, pais e outros) em buscar soluções para o desafio da presença de tão diferentes alunos nas classes, como também do desejo de fazer de tudo para que nenhum aluno, deficiente ou nãodeficiente, seja excluído com base em alguma especificidade ou necessidade que a escola não consegue atentar-se ou dar-se conta.

Inclusão Digital é a democratização do acesso às tecnologias da informação, de forma a permitir a inserção de todos na sociedade da informação. Entre as estratégias inclusivas estão projetos e ações que facilitam o acesso de pessoas de baixa renda às Tecnologias da Informação e Comunicação (TIC). A inclusão digital volta-se também para o desenvolvimento de tecnologias que ampliem a acessibilidade para usuários com deficiência.

Dessa forma, toda a sociedade pode ter acesso a informações disponíveis na Internet, e assim produzir e disseminar conhecimento. A inclusão digital insere-se no movimento maior de inclusão social, um dos grandes objetivos compartilhados por diversos governos ao redor do mundo nas últimas décadas. 
A inclusão digital, a produção e o compartilhamento do conhecimento são de suma importância para o desenvolvimento econômico, cultural, social e político do País (Martins, 2003). De acordo com o autor, cerca de 150 milhões de brasileiros não entraram na era digital. Para Silveira (2005), o domínio das tecnologias da informação e comunicação (TICs) por poucos e a situação de exclusão digital em que se encontram as populações carentes em todo o território nacional contribuem para o aprofundamento das desigualdades econômicas e sociais já tão graves em nossa sociedade.

Para que o dEficiente visual possa sentir-se incluído digitalmente é necessário que tenha recursos de acessibilidade. Acessibilidade significa não apenas permitir que pessoas com deficiências participem de atividades que incluem o uso de produtos, serviços, informação e tecnologias, mas a inclusão e extensão do uso destes por todas as parcelas presentes em uma determinada população. Em informática, programas que provêm acessibilidade são ferramentas ou conjuntos de ferramentas que permitem que portadores de deficiências (as mais variadas) se utilizem dos recursos que o computador oferece.

\section{Softwares leitores de telas disponíveis no mercado}

Atualmente existem diferentes softwares leitores de telas que usam um sintetizador de voz que permite a leitura de informações exibidas na tela de um computador.

Este artigo apresenta os seguintes softwares leitores de telas: Virtual Vision 5.0, Jaws 8.0 e NVDA.

\subsection{VIRTUAL VISION 5.0}

Virtual Vision 5.0 é o programa que permite aos deficientes visuais utilizar o ambiente Windows, os aplicativos Office, e navegar pela Internet com o Internet Explorer. O Virtual Vision 5.0 utiliza o DeltaTalk, a tecnologia de síntese de voz desenvolvida pela MicroPower®, garantindo a qualidade do áudio como o melhor sintetizador de voz em português do mundo.

Há duas versões do Virtual Vision. A versão 2.0, utilizada para Windows $98 \mathrm{e}$ pacote office 97, bem como a versão 5.0, desenvolvida para o sistema operacional Windows XP e pacote office XP ou 2000 com significativas melhorias de navegação na WEB utilizando o Internet Explorer.

\subsection{JAWS 8.0}

JAWS 8.0 oferece tecnologia de voz sintetizada em ambiente Windows para acessar softwares, aplicativos e recursos na internet. Um sintetizador de voz integrado ao software, utiliza a placa e as caixas de som do computador para dar as informações exibidas no monitor. Possibilita também o envio dessa mesma informação a linhas Braille. Esta tecnologia oferece acesso a um leque muito amplo de aplicativos sem o uso do monitor ou do mouse. Também acesso a informações, a educação e ao trabalho.

Durante a instalação falada, o software guia-o em todos os passos, permitindo a seleção entre os vários idiomas (inclusive português falado no Brasil) do sintetizador, que funciona com a maioria de placas de som do mercado.

O JAWS 8.0.1163 é a primeira versão beta deste leitor capaz de se executar e instalar nos computadores com o Windows Vista.

\subsection{NONVISUAL DESKTOP ACCESS (NVDA)}

O NVDA é um leitor de telas para o sistema operacional Windows. Atualmente o NVDA suporta os sintetizadores de voz compatíveis e usa uma estrutura modular, que permite suportar outros sintetizadores de voz que sejam programados. O NVDA é um programa experimental e está em constante desenvolvimento. É de código aberto, isto significa que qualquer pessoa pode ver o código fonte ou mudá-lo a seu gosto para satisfazer os seus interesses e necessidades. 
Para além das funcionalidades gerais do Windows, o NVDA funciona com o WordPad, o bloco de notas,o outlook express e o internet explorer. Também tem suporte básico para o Microsoft Word 2000/xp/2003 e para o Microsoft excel 2000/xp/2003. O NVDA também tem extensões para o Mozilla Firefox 2.0 e superiores, sendo o explorador recomendado para obter maior acessibilidade com este programa. O NVDA roda no Windows XP, Windows Vista e Windows 2000, porém não funcionará no Windows 95/98/me, nem trabalha ainda em versões do Windows de 64 bits.

\section{Qualidade de software}

Este artigo apresenta a avaliação da Qualidade dos softwares leitores de telas apresentados acima, onde cinco usuários dEficientes visuais, que já conhecem ao menos um leitor de telas irão realizar a avaliação dos mesmos. A avaliação da qualidade dos softwares leitores de telas será baseada no modelo de qualidade de software NBR ISO/IEC 9126 (Qualidade do produto de software).

Os computadores têm sido usados numa variedade de áreas de aplicação cada vez maior e sua correta operação é freqüentemente crítica para o sucesso de negócios e para a segurança humana. Deste modo, desenvolver ou selecionar produtos de software de alta qualidade é de primordial importância. Especificação e avaliação da qualidade do produto de software são fatores chave para garantir qualidade adequada. Isto pode ser alcançado pela definição apropriada das características de qualidade, levando em consideração o uso pretendido do produto de software. É importante que cada característica relevante de qualidade do produto de software seja especificada e avaliada utilizando, quando possível, métricas validadas ou amplamente aceitas.

A NBR 13596 - Tecnologia de informação - Avaliação de produto de software Características de qualidade e diretrizes para o seu uso, que foi desenvolvida para suportar estas necessidades, definiu seis características de qualidade e descreveu um modelo de processo para avaliação de produto de software. Como as características de qualidade e as métricas associadas podem ser úteis não só à avaliação de produto de software, mas também para a definição de requisitos de qualidade e outros usos, a NBR 13596 está sendo substituída por duas séries de normas relacionadas: NBR ISO/IEC 9126 (Qualidade do produto de software) e NBR ISO/IEC 14598 (Avaliação de produto de software). As características de qualidade do produto de software definidas nesta parte da NBR ISO/IEC 9126 podem ser usadas para especificar requisitos funcionais e não-funcionais do cliente e do usuário.

É recomendado que, para a avaliação de qualidade de um produto de software, seja definido um modelo de qualidade e que este modelo de qualidade seja usado na definição das metas de qualidade para os produtos de software final e intermediários. Convém que a qualidade do produto de software seja decomposta hierarquicamente em um modelo composto de características e subcaracterísticas, as quais podem ser usadas como uma lista de verificação de tópicos relacionados com a qualidade.

$\mathrm{O}$ modelo de qualidade de software categoriza seus atributos em seis características (funcionalidade, confiabilidade, usabilidade, eficiência, manutenibilidade e portabilidade) as quais são, por sua vez, subdivididas em subcaracterísticas, conforme a figura abaixo. 


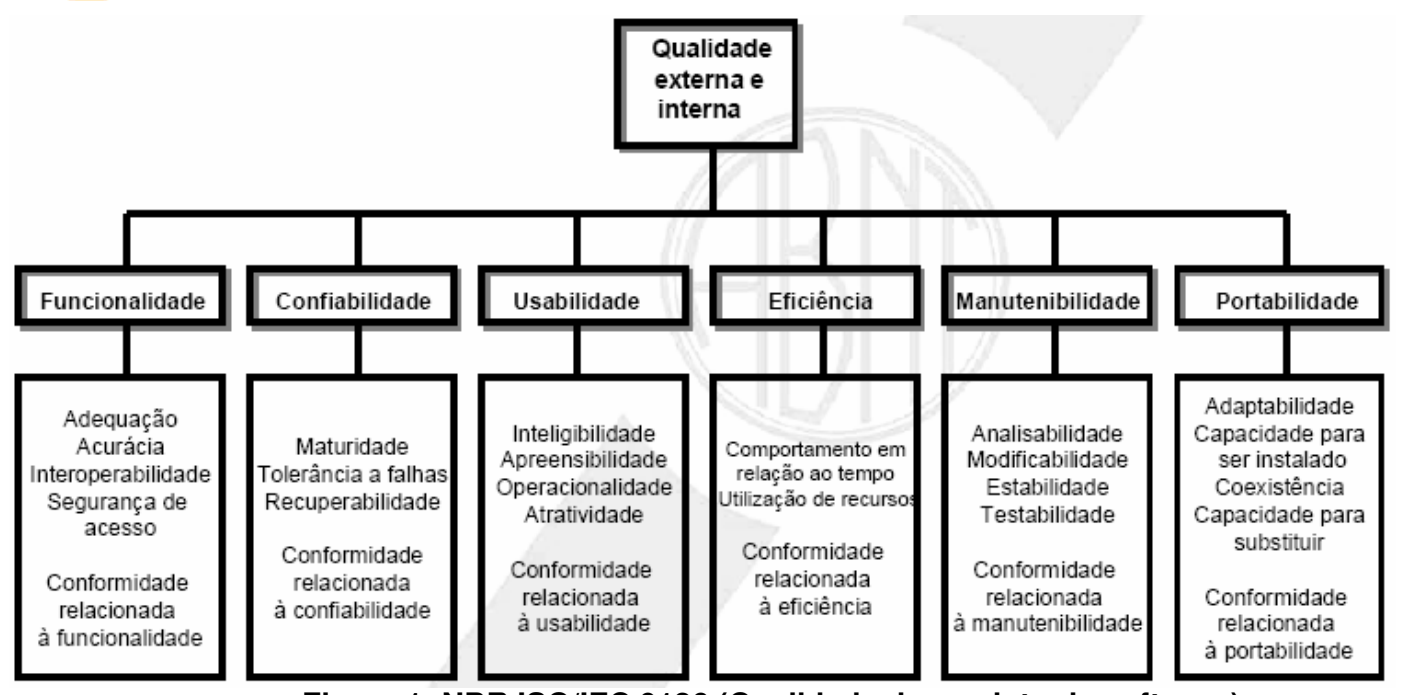

Figura 1: NBR ISO/IEC 9126 (Qualidade do produto de software)

Para realizar a avaliação da qualidade de um software, não precisamos necessariamente avaliar todas as características e suas subcaracterísticas. Podemos, diante do modelo de qualidade de software, escolher simplesmente apenas uma característica e uma de suas subcaracterísticas.

Diante do modelo de qualidade de software apresentado acima, neste estudo entende-se como pertinente para a avaliação dos softwares leitores de telas a característica Funcionalidade e suas subcaracterísticas: Acurácia (Accuracy), Conformidade; a característica Usabilidade e suas subcaracterísticas: Inteligibilidade, Apreensibilidade, Operacionalidade; e a característica Eficiência e sua subcaracterística: Comportamento com relação ao uso de recursos.

Como o teste foi realizado por cinco usuários dEficientes visuais, somente as características e subcaracterísticas citadas no parágrafo anterior foram relevantes para a avaliação da qualidade dos softwares leitores de telas Virtual Vision, Jaws e NVDA, uma vez que estaremos avaliando a nível de usuário. Sendo assim, nem todas as características e subcaracterísticas apresentadas no modelo de qualidade de software são importantes.

Segue, abaixo, a descrição das características, a descrição de suas subcaracterísticas, bem como a pergunta relacionada elaborada para a realização da avaliação do software leitor de telas.

a) Característica Funcionalidade: Capacidade do produto de software de prover funções que atendam às necessidades explícitas e implícitas, quando o software estiver sendo utilizado sob condições especificadas.

a.1) Subcaracterística Acurácia (Accuracy): Capacidade do produto de software de prover, com o grau de precisão necessário, resultados ou efeitos corretos ou conforme acordados.

Pergunta: O software tem um bom sintetizador de voz, de forma que ao ler as telas o usuário entenda o que o software está falando?

a.2) Subcaracterística Conformidade: Capacidade do produto de software de estar de acordo com normas, convenções ou regulamentações previstas em leis e prescrições similares relacionadas à funcionalidade.

Pergunta: Os comandos e atalhos utilizados no Windows e pacote Office permanecem os mesmos enquanto o software leitor de tela está operando?

b) Característica Usabilidade: Capacidade do produto de software de ser compreendido, aprendido, operado e atraente ao usuário, quando usado sob condições especificadas. 
b.1) Subcaracterística Inteligibilidade: Capacidade do produto de software de possibilitar ao usuário compreender se o software é apropriado e como ele pode ser usado para tarefas e condições de uso específicas.

Pergunta: O software leitor de telas proporciona a mesma lógica de funcionamento do Windows, ou seja, quando pressionado o botão iniciar, fala se o item navegado é um menu, submenu, atalho ou caixas de diálogo?

b.2) Subcaracterística Apreensibilidade: Capacidade do produto de software de possibilitar ao usuário aprender sua aplicação.

Pergunta: O deficiente visual terá o mesmo raciocínio de operabilidade utilizando o software leitor de telas para utilizar o sistema operacional windows? Ex.:

Usar os comandos alt+f4 para fechar as janelas, utilizar a tecla tab para navegar nas caixas de diálogo do Windows.

b.3) Subcaracterística Operacionalidade: Capacidade do produto de software de possibilitar ao usuário operá-lo e controlá-lo.

Pergunta: Como você considera o leitor de telas quanto à facilidade de uso?

c) Característica Eficiência: Capacidade do produto de software de apresentar desempenho apropriado, relativo à quantidade de recursos usados, sob condições especificadas.

c.1) Subcaracterística Comportamento com relação ao uso de recursos: Capacidade do produto de software de usar tipos e quantidades apropriados de recursos, quando o software executa suas funções sob condições estabelecidas.

Pergunta: O software informa quais são as medidas da quantidade de recursos necessários (CPU, disco e memória, dentre outros) e o tempo aproximado de instalação para o deficiente visual instalar?

Para o questionário com avaliação dos softwares leitores de telas, cada resposta terá a opção de Satisfatório, Parcialmente satisfatório e Insatisfatório.

Em uma escala de 1 à 3, o artigo define peso 3 para a resposta de satisfatório, peso 2 para parcialmente satisfatório e peso 1 para insatisfatório.

\section{Forma de avaliação e aplicação do software}

Conforme dito anteriormente, os softwares leitores de telas foram avaliados por cinco usuários dEficientes visuais de três formas diferentes. Dois usuários realizaram a avaliação em um laboratório de informática de uma determinada escola. Outros dois usuários avaliaram os softwares em sua residência. $\mathrm{O}$ quinto usuário realizou o teste a distância, ou seja, foi enviado e recebido o questionário por email, onde o usuário realizou o teste em sua residência. Isso só foi permitido porque trata-se de um usuário dEficiente visual avançado que já utiliza softwares leitores de telas há seis anos.

Cada usuário dEficiente visual navegou em um software leitor de telas de cada vez, realizando uma navegação pelo sistema operacional Windows XP onde foram explorados alguns aplicativos mais básicos como bloco de notas, calculadora além de aplicativos do pacote Microsoft Office. No Microsoft Word, por exemplo, os usuários digitaram um pequeno texto, realizaram edições como apagar e adicionar texto, acessar a barra de menus, salvar, abrir, fechar.

Além disso, individualmente cada usuário dEficiente visual tentou, durante a navegação, identificar se compreendiam a leitura e a lógica do sistema operacional Windows como por exemplo itens como menu, submenu, bem como as caixas de diálogo de alguns aplicativos como o bloco de notas e Microsoft Word que questionam quanto ao desejo de salvar o conteúdo digitado.

Durante a navegação e exploração do sistema operacional junto ao software leitor de tela que estava sendo avaliado, o questionário impresso era lido e marcado por uma pessoa vidente responsável pela tabulação dos dados avaliados.

A tabela abaixo apresenta os resultados desta avaliação. 
RESULTADO AVALIAÇÃO SOFTWARES LEITORES DE TELA:

\begin{tabular}{|c|c|c|c|c|}
\hline \multirow[b]{2}{*}{ DESCRIÇÃO DA CARACTERÍSTICA } & \multirow[b]{2}{*}{ Peso } & \multicolumn{3}{|c|}{ Notas } \\
\hline & & $\begin{array}{c}\text { Virtual } \\
\text { Vision } 5.0\end{array}$ & Jaws 8 & NVDA \\
\hline $\begin{array}{l}\text { Característica 1. USABILIDADE } \\
1.1 \text { Inteligibilidade } \\
1.2 \text { Apreensibilidade } \\
1.3 \text { Operacionalidade }\end{array}$ & $\begin{array}{l}4,0 \\
4,0 \\
3,0 \\
3,0\end{array}$ & $\begin{array}{l}3,0 \\
3,0 \\
3,0 \\
3,0\end{array}$ & $\begin{array}{l}3,0 \\
3,0 \\
3,0 \\
\mathbf{3}, 0\end{array}$ & $\begin{array}{l}2,6 \\
3,0 \\
2,6 \\
\mathbf{2 , 7}\end{array}$ \\
\hline $\begin{array}{l}\text { Característica 2. FUNCIONALIDADE } \\
\text { 2.1 Acurácia } \\
2.3 \text { Conformidade } \\
\end{array}$ & $\begin{array}{l}4,0 \\
5,0 \\
5,0\end{array}$ & $\begin{array}{l}2,8 \\
3,0 \\
2,9\end{array}$ & $\begin{array}{l}2,8 \\
3,0 \\
2,9\end{array}$ & $\begin{array}{l}2,2 \\
3,0 \\
2,6\end{array}$ \\
\hline $\begin{array}{l}\text { Característica 3. EFICIÉNCIA } \\
3.1 \text { Comportamento em relaçẫo ao tempo } \\
\text { Média indicador }\end{array}$ & $\begin{array}{l}2,0 \\
10,0\end{array}$ & $\begin{array}{l}2,4 \\
\mathbf{2 , 4}\end{array}$ & $\begin{array}{l}2,2 \\
\mathbf{2 , 2}\end{array}$ & $\begin{array}{l}1,4 \\
\mathbf{1 , 4}\end{array}$ \\
\hline Média Geral: & 10,0 & 2,84 & 2,80 & 2,41 \\
\hline
\end{tabular}

Tabela 1. Resultado da avaliação dos softwares leitores de tela:

Representaremos graficamente abaixo o resultado da avaliação das três características Usabilidade, Funcionalidade e Eficiência avaliados pelos cinco usuários dEficientes visuais.

A figura 3 apresenta a média da característica Usabilidade bem como suas subcaracterísticas Inteligibilidade, Apreensibilidade e Operacionalidade. Foi possível verificar que os resultados dos softwares Virtual Vision e Jaws foram os mesmos, uma vez que os leitores oferecem praticamente os mesmos recursos quanto a usabilidade. O NVDA ficou com 2,7 devido há alguns avaliadores acharem a facilidade de uso parcialmente satisfatória, uma vez que o sintetizador de voz fala rápido por padrão, apesar disso, podemos configurar a velocidade de fala do sintetizador em seu painel de controle.

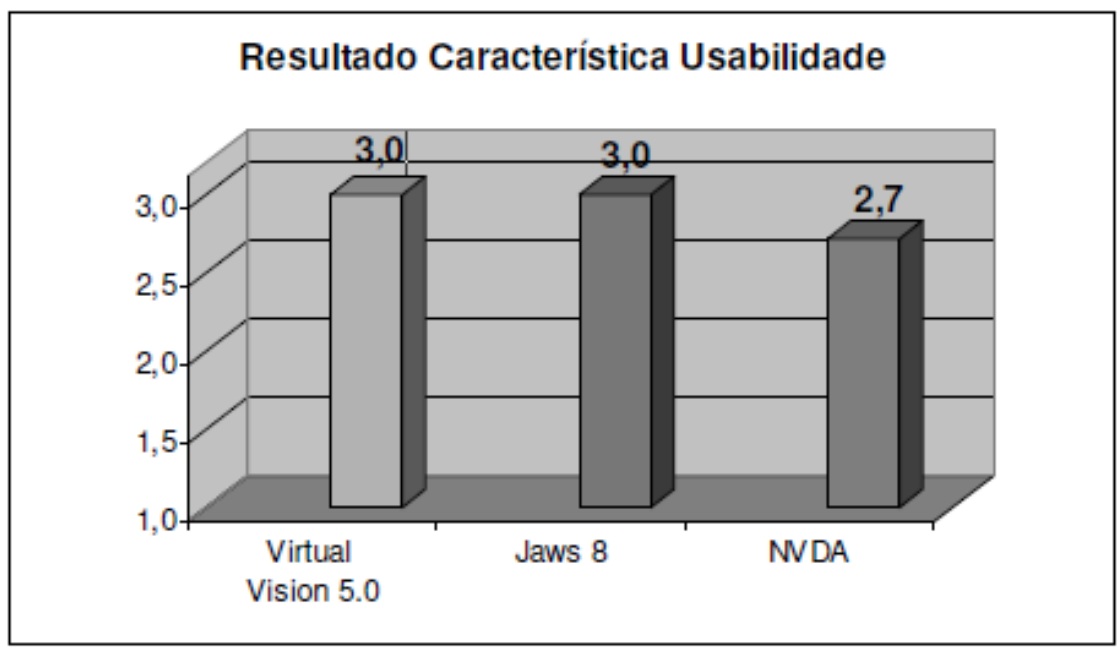

Figura 3: Gráfico resultado característica Usabilidade.

$\mathrm{Na}$ característica Funcionalidade (figura 4), pode-se perceber que a funcionalidade do Virtual Vision 5.0 e Jaws 8 foram as mesmas, onde podemos novamente afirmar que as característica de Funcionalidade de ambos os softwares são muito parecidas. O NVDA 
ficou com 2,6 devido a dificuldade de alguns avaliadores entenderem o que o software está falando, bem como em algumas situações o NVDA não lê exatamente o que está na tela, confundindo o usuário dEficiente visual.

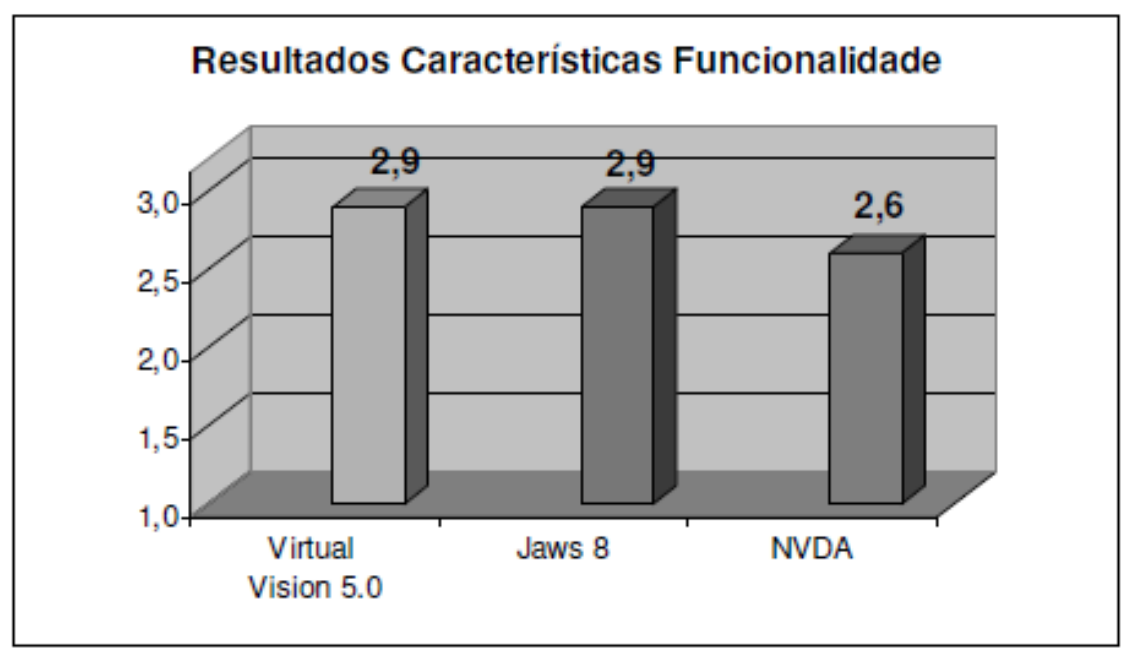

Figura 4: Gráfico resultado característica Funcionalidade.

Na figura 5, podemos notar que os usuários dEficientes visuais ainda não estão completamente satisfeitos na característica de Eficiência. O Virtual Vision oferece um CD de instalação que permite ao usuário dEficiente visual instalar o software sozinho, oferecendo ao usuários todas as informações necessárias para a instalação bem como quais as medidas da quantidade de recursos necessários. O jaws oferece todas as informações em seu site, onde é possível obter essas informações, mas precisa que um vidente faça isso a ele. O NVDA não oferece um suporte de instalação, tem que ser baixado na Web, onde o dEficiente visual precisará da ajuda de uma pessoa vidente.

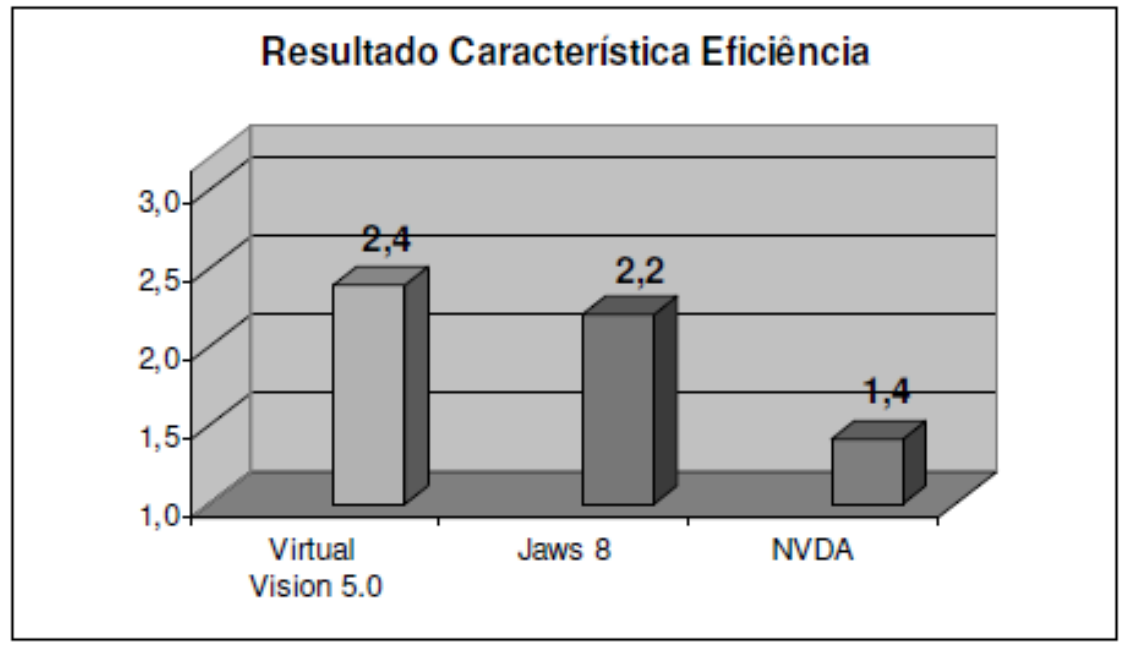

Figura 5: Gráfico resultado característica Eficiência

\subsection{Considerações finais}

O presente artigo mostrou que atualmente temos muitas tecnologias de software para a inclusão de dEficientes visuais, mas a acessibilidade a esses softwares continuam sendo a realidade de poucas pessoas. Com nossos avaliadores dEficientes visuais, por exemplo, dois deles realizaram o teste do software em uma escola que emprestou seu 
laboratório para realizarmos as avaliações, mas isso, por que são incluídos, não somente na tecnologia de software, mas também são incluídos na sociedade, realizaram cursos de orientação e mobilidade e conseguem se locomover sozinhos, além de fazer a maiorias das atividades que qualquer outra pessoa vidente conseguiria. Outros dois alunos, não foram a essa determinada escola porque seu pai não pode lhes levar na escola naquele dado momento, ou seja, a superproteção dos pais os impediu de sua inclusão social, portanto, o avaliador teve que ir até suas casas para realizar a avaliação dos softwares.

Já o quinto usuário que realizou a avaliação do software já é uma pessoa mais experiente, sabe andar sozinho pelas ruas, pegar ônibus, ir de uma cidade a outra, conhece, estuda e explora diversos softwares leitores de telas, por isso, o mesmo realizou a avaliação dos softwares a distância.

Portanto, apenas com cinco exemplos, tivemos realidades bem diferentes, o que mostra a importância da inclusão digital bem como a importância da acessibilidade para permitir que pessoas com deficiência participem de atividades que incluem o uso de produtos, serviços, informação e tecnologia.

Diante de importância da acessibilidade de pessoas com deficiência visual, concluímos que realizar uma avaliação utilizando um modelo de qualidade de software NBR ISO/IEC 9126 e aplicar ao menos a cinco usuários dEficientes visuais, podemos ter uma avaliação verdadeira e precisa dessas tecnologias, uma vez que esses avaliadores são os próprios usuários que utilizam essas tecnologias inclusivas.

No resultado final de nossa avaliação, o Virtual Vision 5.0 atendeu em 2,84 aos quesitos de qualidades propostos; o Jaws atendeu aos mesmos quesitos de qualidades em 2,8 e o novo e pouco conhecido NVDA atenteu aos quesitos de qualidade em 2,41.

Concluímos em nosso artigo que o virtual vision 5.0 tem um sintetizador melhor, ou seja, fala de forma mais clara e entendível em relação ao Jaws 8 que oferece sintetizador que fala um português "americanizado". O que realmente nos surpreendeu, foi o NVDA, que apesar de muito novo, atendeu aos requisitos de qualidade de software avaliados. Mesmo ficando atrás do Virtual Vision e do Jaws, o NVDA sintetizador de voz do windows, que por sua vez é americanizado e não conseguimos entender bem o que ele fala. Por outro lado, o NVDA permite a instalação de outros sintetizadores de voz, onde podemos ter uma ótima leitura das telas.

Finalmente, o este artigo comprovou através da avaliação da qualidade de software, que uma vez que uma pessoa com dEficiência visual, conheca ao menos um software leitor de telas, o mesmo conseguirá utilizar outros leitores de tela, afinal, apesar dos mesmos apresentarem fabricantes diferentes, oferecem o mesmo raciocínio de operação, mudando apenas alguns detalhes durante a fala que não fazem grandes diferenças, ficando assim ao gosto e critério de cada usuário dEficiente visual.

Neste artigo, apresenta-se a avaliação de qualidade de software para apenas três leitores de telas, entretanto, existem outros softwares leitores de telas no mercado, como o dosvox, por exemplo que é bastante conhecido e explorado por seu público. Além disso, estão surgindo e irão surgir novas tecnologias para facilitar a inclusão digital.

Para trabalhos futuros, podemos citar novas tecnologias de softwares leitores de telas para sistema operacional Linux, onde não teremos o acesso gratuito somente do software leitor de telas, mas também um sistema Operacional Linux. Além disso, novas tecnologias de softwares leitores de telas para aparelhos de celulares a um preço de custo mais popular e acessível a toda população.

\section{Bibliografia:}

Beyer, H. O. Inclusão e Avaliação na Escola. Porto Alegre: Mediação, 2005. 164p. Bruno, Marilda Moraes Garcia (1997). Deficiência Visual: Reflexão sobre a prática pedagógica. São Paulo: Laramara.

Carvalho, Marco Aurélio (2006). "XVII Simpósio Brasileiro de Informática na 
Educação. XVII 2006. Brasília-DF.”, Educação a distância e inclusão digital: ações para a cidadania e o desenvolvimento social: Moreira, Ana Cristina S. Moreira, Moretti, Telma, p. 145-152.

Escola e cidadania: o papel da escola na formação para dewmocracia. Porto Alegre: Artmed, 2005. 184p.

Heidrich, R.O. Análise de processo de inclusão escolar de alunos com paralisia cerebral utilizando as tecnologias de informação e comunicação, no estado do Rio Grande do Sul. Porto Alegre, RS, 2004. 230. Tese de Doutorado em Informática na Educação.

Perrenoud, P. Ensinar: agir na urgência, decidir na incerteza. Porto Alegre: Artmed, 2001, 208p.

Software leitor de telas Jaws. Disponível em <http://www.freedomscientific.com>, Acesso em : 17 jun 2007.

Software leitor de telas NVDA. Disponível em <http://www.nvda-project.org/>, Acessado em: 14 mai 2007.

Software leitor de telas Virtual Vision. Disponível em < http://www.micropower.com.br >. Acessado em 17 jun 2007. 